
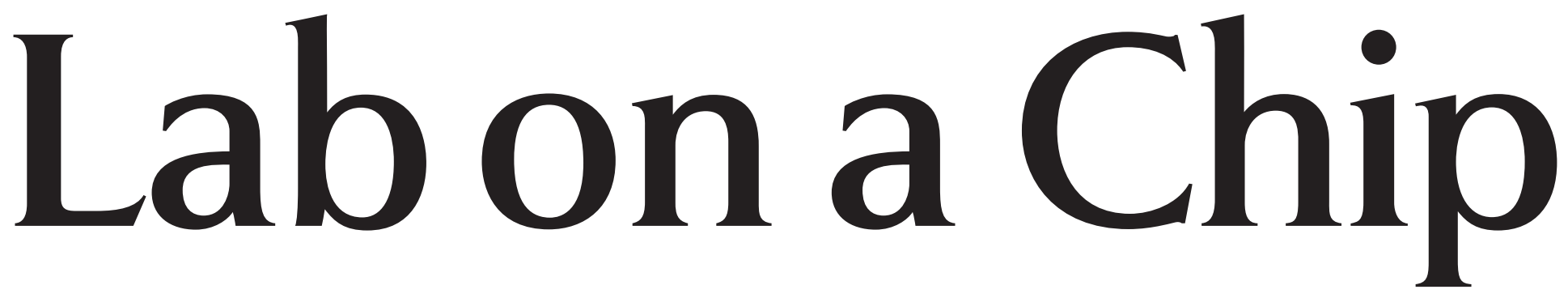

Miniaturisation for chemistry, physics, biology, materials science and bioengineering

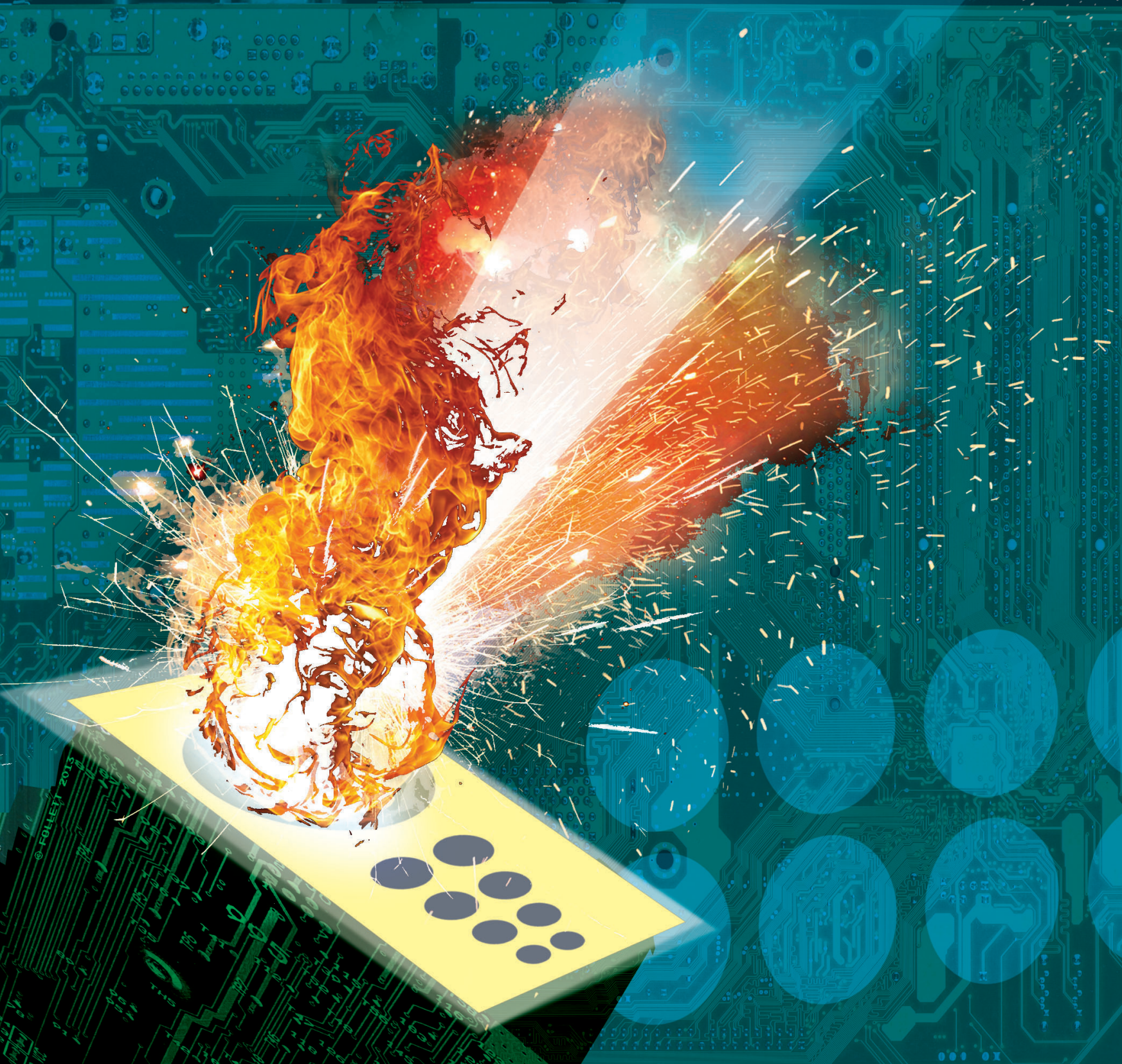


Cite this: Lab Chip, 2013, 13, 4164

\title{
A portable explosive detector based on fluorescence quenching of pyrene deposited on coloured wax- printed $\mu$ PADs
}

\author{
Regina Verena Taudte, ${ }^{a}$ Alison Beavis, ${ }^{a}$ Linzi Wilson-Wilde, ${ }^{b}$ Claude Roux, ${ }^{a}$ \\ Philip Doble a and Lucas Blanes*a
}

\begin{abstract}
A new technique for the detection of explosives has been developed based on fluorescence quenching of pyrene on paper-based analytical devices ( $\mu$ PADs). Wax barriers were generated $\left(150{ }^{\circ} \mathrm{C}, 5 \mathrm{~min}\right)$ using ten different colours. Magenta was found as the most suitable wax colour for the generation of the hydrophobic barriers with a nominal width of $120 \mu \mathrm{m}$ resulting in fully functioning hydrophobic barriers. One microliter of $0.5 \mathrm{mg} \mathrm{mL}^{-1}$ pyrene dissolved in an $80: 20$ methanol-water solution was deposited on the hydrophobic circle ( $5 \mathrm{~mm}$ diameter) to produce the active microchip device. Under ultra-violet (UV) illumination, ten different organic explosives were detected using the $\mu P A D$, with limits of detection ranging from 100-600 ppm. A prototype of a portable battery operated instrument using a $3 \mathrm{~W}$ power UV light-emitting-diode (LED) (365 nm) and a photodiode sensor was also built and evaluated for the successful automatic detection of explosives and potential application for field-based screening.
\end{abstract}

Received 17th May 2013,

Accepted 31st July 2013

DOI: $10.1039 / \mathrm{c} 31 \mathrm{c} 50609 f$

www.rsc.org/loc addition, sending samples for laboratory testing is costly and time-consuming. ${ }^{4}$

It is therefore of significance to the research community to develop portable screening methods to detect explosives. Some mature techniques include amperometric sensing, ${ }^{18}$ polymer sensing, ${ }^{17,19}$ membrane-based flow displacement immunoassays, ${ }^{20-24}$ amperometric gas sensoring, ${ }^{18}$ reflectance spectroscopy $^{3}$ and colour reactions. ${ }^{25,26}$ Although these methods facilitate field detection of explosives, they can be time intensive, have significant sample preparation requirements or include elaborate screening devices which may not be stable under general or non-laboratory conditions. ${ }^{3}$

Fluorescence methods have also been applied to the field detection of explosives due to their sensitivity, selectivity, simplicity and low cost instrumentation. ${ }^{27-31}$ Polymer films have been used as fluorophore sensors, however, these films are difficult to synthesise and are mostly constrained to the detection of explosives in an aqueous solution..$^{30,31}$

Various "Lab-on-a-chip" devices, particularly CE-based electrochemical microsystems, have also been used in the field of explosive detection. ${ }^{7}$ These microsystems are advantageous due to their portability, compact size, relatively easy integration with other systems, low cost and low sample/ reagent consumption, and have rapid analysis times. ${ }^{7}$

Microfluidic paper-based analytical devices ( $\mu$ PADs) present a new generation/class in microfluidics. Filter paper is used as a fabrication substrate on which hydrophobic microfluidic patterns are imparted to control fluid movement and compartmentalise chemical reactions. Various methods may 
be employed to construct $\mu$ PADs including photolithography, ${ }^{32}$ plotting, ${ }^{33}$ paper cutting, ${ }^{34}$ plasma oxidation, ${ }^{35}$ inkjet printing, ${ }^{36}$ inkjet etching ${ }^{37,38}$ and wax printing. ${ }^{39-41}$ In comparison to other micro devices, $\mu$ PADs have particular benefits such as, no requirement for pumps, adsorption of aqueous fluids, availability in a range of thickness, easy disposal, flexibility, and suitability for colorimetric tests. ${ }^{42}$

In this paper we present an innovative wax printed $\mu \mathrm{PAD}$ and prototype instrument to detect explosives using an ultraviolet LED.

\section{Materials and methods}

\section{Chemicals and reagents}

Terasil Blue, Orange G and Acid Green were obtained from Sigma Aldrich in analytical grade. Pyrene and sodium dodecyl sulfate (SDS) were used in analytical grade. Explosives, i.e. 1,3,5-trinitrobenzene (TNB), 2,4,6-trinitrotoluene (TNT), 2,4dinitrotoluene (2,4-DNT), 4-amino-2,6-dinitrotoluene (4-A-2,6DNT), 4-nitrotoluene (4-NT), 1,3-dinitrobenzene (DNB), nitrobenzene (NB), 1,3,5-trinitro-1,3,5-triazacyclohexane (RDX), pentaerythritol tetranitrate (PETN), and 2,4,6-trinitrophenylmethylnitramine (tetryl), at a certified concentration of 1000 $\mu \mathrm{g} \mathrm{mL}^{-1}$ in acetonitrile, were obtained from ChemService (West Chester, PA, USA).

Ultra pure water $\left(18.2 \mathrm{M} \Omega \mathrm{cm}^{-1}\right)$ was used throughout all experiments and obtained from a Sartorius 611 water purification system. Methanol, ethanol, propanol, hexane and acetonitrile were analytical grade (Sigma-Aldrich, Australia). Coffee, tea, Coca Cola ${ }^{\mathrm{TM}}$, beer, wine, milk and Mylanta Antacid Dual Action, a stomach antacid, were purchased at Australian supermarkets.

Colour solutions were obtained by dissolving $1 \mathrm{mg}$ of Terasil Blue, Orange $\mathrm{G}$ and Acid Green each in $1 \mathrm{~mL}$ water.

A pyrene solution was prepared by dissolving $2.5 \mathrm{mg}$ of pyrene in $4 \mathrm{~mL}$ methanol. This solution was sonicated for five minutes followed by the addition of $1 \mathrm{~mL}$ deionised water producing a $0.5 \mathrm{mg} \mathrm{mL}^{-1}$ pyrene solution.

Great care has to be taken when handling explosive materials. All explosives were stored in an explosion proof fridge $\left(4{ }^{\circ} \mathrm{C}\right)$ securely sealed in appropriate glass containers. Explosive solutions were prepared and experiments conducted in well ventilated fume hoods wearing appropriate personal protection, i.e. face protection, gloves, and lab coat.

\section{$\mu \mathrm{PAD}$ fabrication}

CorelDraw 5 software (Corel Corporation, Ontario, Canada) was used to design the microstructures of the $\mu \mathrm{PAD}$ prior to printing the designs on Whatman ${ }^{\circledR} 185 \mathrm{~mm}$ diameter filter paper grade 40:8 $\mu \mathrm{m}$ (Ashless Grade, Ash 0.007\%). The $\mu$ PADs were printed with a FujiXerox ColorQube 8870 colour printer (Xerox, Australia). The printed wax channels were melted (at $150{ }^{\circ} \mathrm{C}$ for $5 \mathrm{~min}$ ) into the filter paper with a swing-away heat press (GEO Knight \& Co, Inc) with pressure and temperature control. An EZ4D microscope (Leica) was used to measure the width of the wax lines.
The channel width selected in the software was referred as nominal width $\left(W_{\mathrm{N}}\right)$, the width after printing as printed width $\left(W_{\mathrm{P}}\right)$ and the width after heating as $W_{\mathrm{H}} \cdot{ }^{39}$

Wax barrier optimisation. Lines of varying widths and colours were printed to evaluate the extent of spreading when heated at $150{ }^{\circ} \mathrm{C}$ for $5 \mathrm{~min}$. The lines' $W_{\mathrm{N}}$ ranged from $100 \mu \mathrm{m}$ to $1000 \mu \mathrm{m}$ with increments of $100 \mu \mathrm{m}$. All lines were designed and printed in all colours available on the printer; cyan, magenta, yellow and black (colours present the CMYK colour model) and green as a mixed colour. The spreading of the different colours was examined by measuring $W_{\mathrm{P}}$ and $W_{\mathrm{H}}$ of the lines under the microscope. Hydrophobic barriers were tested by generating circles of different colours with $5 \mathrm{~mm}$ diameter and $W_{\mathrm{N}}$ ranging from 50 to $450 \mu \mathrm{m}$. Five $\mu \mathrm{L}$ of a solution of the Terasil Blue, Orange $G$ and Acid Green solutions were deposited inside the wax barriers. Circles with larger diameters and widths $\left(11 \mathrm{~mm}\right.$ diameter and $\left.600 \mu \mathrm{m} W_{\mathrm{N}}\right)$ were placed around the inner circle in order to stop liquid from spreading over the filter paper (Fig. 5).

Optimised $\mu$ PAD design. After optimisation all $\mu$ PADs were designed with magenta circles of $5 \mathrm{~mm}$ diameter and $200 \mu \mathrm{m}$ nominal width.

\section{Explosive detection by fluorescence quenching}

Solvents for pyrene. Organic solvents (methanol, ethanol, propanol, and acetonitrile) were mixed with water in ratios from 10 : 90 (organic solvent : water) to $100 \%$ organic solvents in increments of $10 \%$. The mixture was coloured with Terasil Blue $\left(1 \mathrm{mg} \mathrm{mL}^{-1}\right)$ and applied to magenta circles of $5 \mathrm{~mm}$ diameter and $200 \mu \mathrm{m} W_{\mathrm{N}}$.

Influence of solvents on wax barriers. The possible influence of organic solvents on the wax barriers was tested by depositing $5 \mu \mathrm{L}$ of hexane and methanol separately in magenta wax circles of $5 \mathrm{~mm}$ diameter and $200 \mu \mathrm{m} W_{\mathrm{N}}$, letting the organic solvents dry and applying organic solvent: water mixtures as in the previous experiment.

\section{Detection}

A Video Spectral Comparator VSC 6000 (Foster + Freeman Ltd, England) was employed for fluorescence detection. The instrument contains a high-resolution colour camera, zoom lens, a range of viewing filters, and multiple illumination sources from UV to visible to IR wavelengths. Fluorescence and fluorescence quenching were visually detected on-screen by exposing the $\mu \mathrm{PAD}$ to the excitation wavelength of $365 \mathrm{~nm}$.

\section{Portable explosive detector prototype}

An in-house prototype $\mu \mathrm{PAD}$ reader device was built based on the electronic circuits shown in Fig. 1. All electronic components were procured from Element 14 (au.elemtn14. com). The UV-LED (365 nm) was a surface mount device type (SMD) with $3 \mathrm{~W}$ power. The sensor was a $5 \mathrm{~mm}$ ambient light photodiode (Vishay, TEPT5700).

Calibration: a $\mu \mathrm{PAD}$ containing only pyrene was inserted between D4 and D1 while R2 was adjusted until the LED D3 turned green and the LED D2 turned off. 


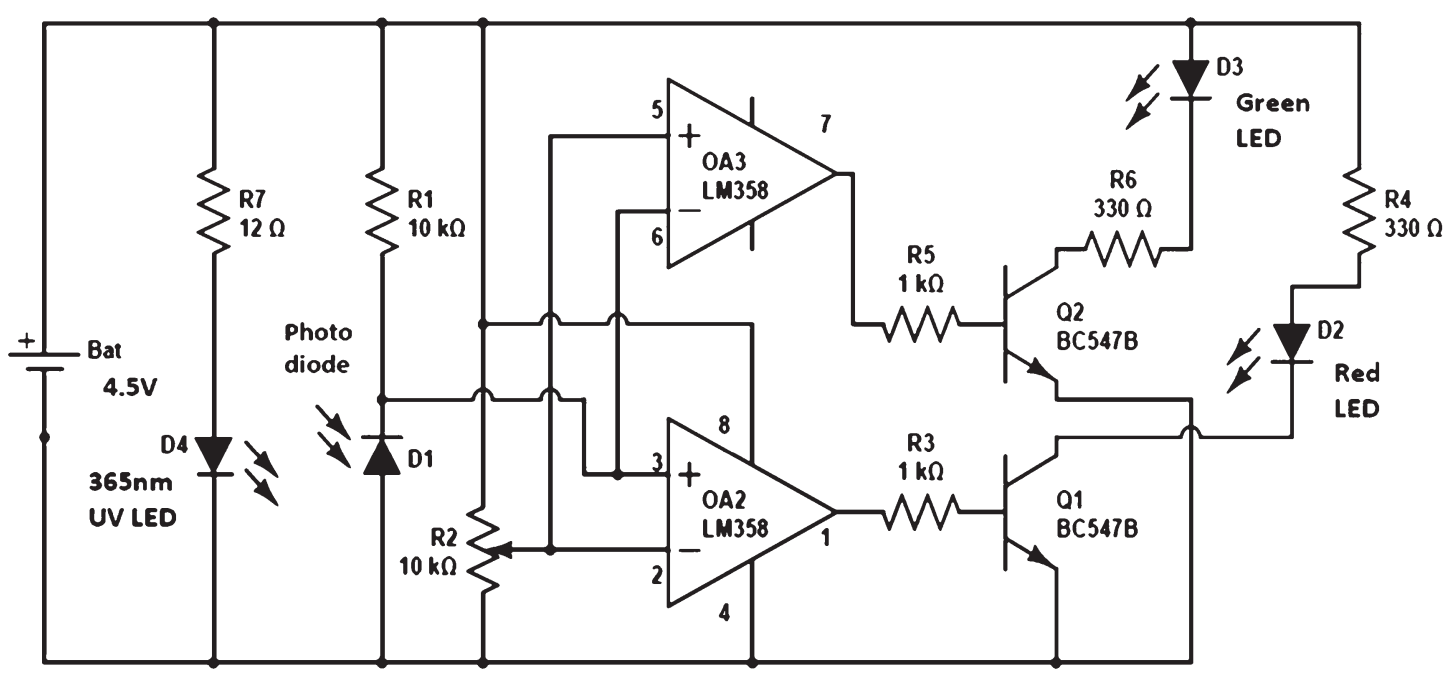

Fig. 1 Electronic diagram of the $\mu$ PAD reader device.

\section{Results and discussion}

\section{$\mu$ PAD fabrication}

Fig. 2 illustrates the process of wax printing in which the wax lines are spread laterally and vertically after controlled heating and pressure application. ${ }^{39}$ Vertical spreading creates the hydrophobic barriers, whilst lateral spreading decreases the sharpness of the channel defined by the barrier and also broadens the width of the barrier. ${ }^{39}$ During the fabrication process fibers in the paper tend to be more horizontally aligned rather than vertically and spreading is quicker along the fibers, the process of lateral spreading is usually more extensive. $^{39}$

The resulting channel width $W_{\mathrm{H}}$ after heating is the sum of the original printed channel width, $W_{\mathrm{P}}$, and two times the distance which the wax spreads from the edge of a printed line, $L$. The spreading characteristics of the wax are likely to be governed by the wax matrix as well as the components which imparts colour. Fig. 3 illustrates the differences in the lateral

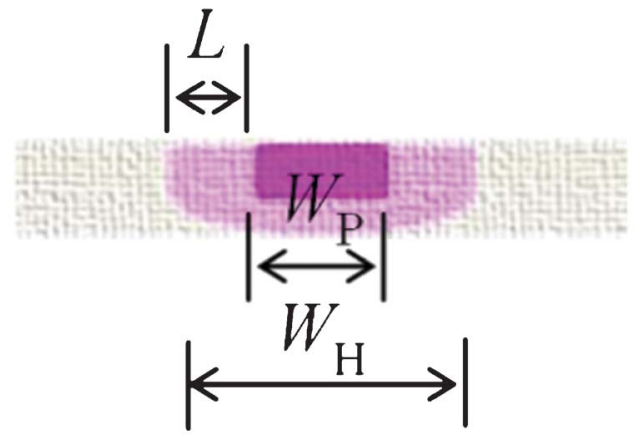

Fig. 2 Schematic illustration of the spreading process of the wax, where $W_{H}=$ $W_{\mathrm{P}}+2 L$ with $W_{\mathrm{H}}$ representing the width after heating, $W_{\mathrm{P}}$ the printed width and $L$ the distance between the spreaded wax and the edge of the printed line, adapted from. ${ }^{39}$ spreading for vertical lines with $0.3 \mathrm{~mm} W_{\mathrm{N}}$ measured after printing (upper line) and after heating (lower line).

The widths of the lines were $0.394 \pm 0.003 \mathrm{~mm}$ before heating and $1.52 \pm 0.04 \mathrm{~mm}$ after heating for yellow, $0.397 \pm$ $0.009 \mathrm{~mm}$ before heating and $1.59 \pm 0.05 \mathrm{~mm}$ after heating for cyan, $0.417 \pm 0.011 \mathrm{~mm}$ before heating and $1.80 \pm 0.02$ $\mathrm{mm}$ after heating for black, $0.458 \pm 0.005 \mathrm{~mm}$ before heating and $2.16 \pm 0.04 \mathrm{~mm}$ after heating for magenta, and $0.454 \pm$ $0.005 \mathrm{~mm}$ before heating and $2.17 \pm 0.03 \mathrm{~mm}$ after heating for green. Yellow displayed the smallest spread, followed by cyan, black, magenta and green. This trend was consistent for every colour from $0.1 \mathrm{~mm}$ to $1.0 \mathrm{~mm} W_{\mathrm{N}}$ with increments of $0.1 \mathrm{~mm}$ (Fig. 4). All colours had linear relationships between heated and nominal width; however with different slopes. It can be seen, that though cyan displayed increased spreading compared to yellow at nominal widths between 0.1 and 0.7 $\mathrm{mm}$, yellow spread more than cyan starting from $0.8 \mathrm{~mm}$ nominal width. The linear relationship indicates that the printing and heating process is reproducible and predictable across these widths.

Thus, it seems possible to design colourful $\mu$ PADs using the FujiXerox ColorQube 8870 colour printer with channels of different predictable widths and characteristics based on the choice of colour.

The quality of hydrophobic barriers was optimised by determining the smallest $W_{\mathrm{N}}$ required for producing fully functioning hydrophobic barriers for every colour present in the four solid inks used in the printer (CMYK colours) plus the colour green representing a mixed colour. Terasil Blue solution (Fig. 5), Orange G solution (data not shown) and Acid Green solution (data not shown) were deposited in circles of varying $W_{\mathrm{N}}$ (from $50-450 \mu \mathrm{m}$ ) and colour (Fig. 5). ${ }^{39}$ The "high" volume of $5 \mu \mathrm{L}$ (forming a convex meniscus on the paper surface) was chosen as it could fully fill the inner and outer circle.

Full functioning barriers were apparent at different widths for the various colours. After heating magenta and green created full hydrophobic barriers with a $W_{\mathrm{N}}$ of $120 \mu \mathrm{m}$, black 


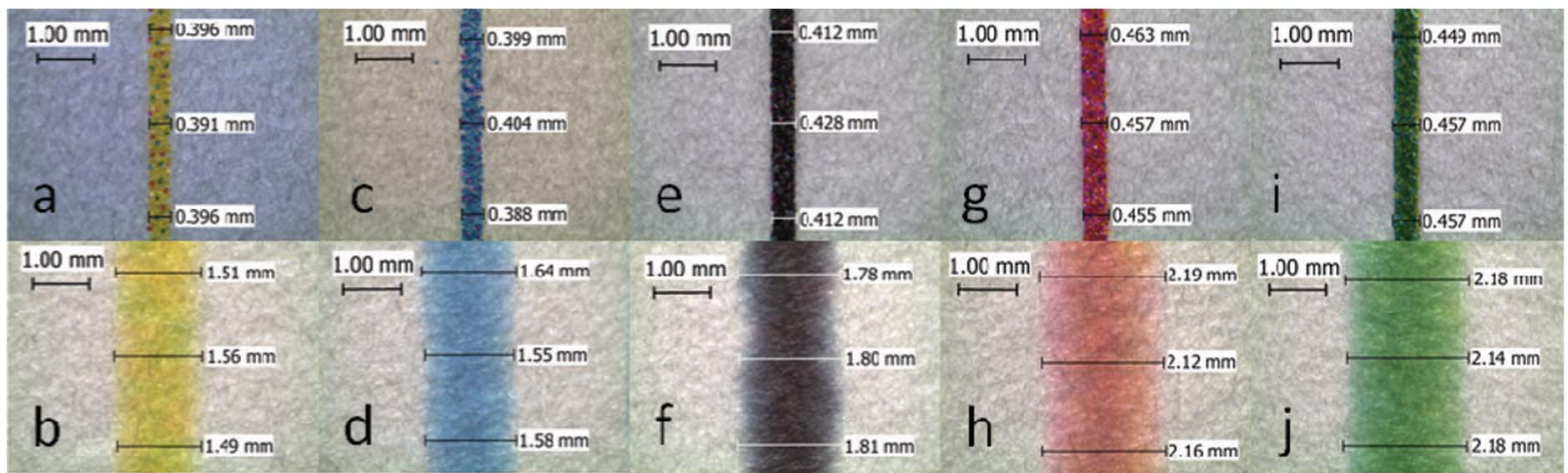

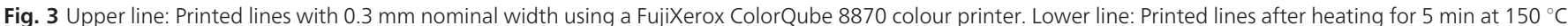
using a swing-away heat press (GEO Knight \& Co, Inc). Colours tested were yellow ( $a, b)$, cyan (c, d), black (e, f), magenta ( $g$, h) and green (i, j). The lines were measured and the pictures taken with a EZ4D microscope (Leica).

with $175 \mu \mathrm{m}$, cyan with $250 \mu \mathrm{m}$, and yellow with $325 \mu \mathrm{m}$. These results are consistent with the trend seen in Fig. 3 and 4 and may be explained by the larger spread of magenta and green in comparison to the other colours upon heating.

A similar procedure was performed with circles of 50-300 $\mu \mathrm{m} W_{\mathrm{N}}$ with increments of $50 \mu \mathrm{m}$ for five mixed colours; orange, dark green, light green, blue and dark purple; and a reference standard, magenta (data not shown). All mixed colours (except light green) produced reliable hydrophobic barriers at a $W_{\mathrm{N}}$ of $130 \mu \mathrm{m}$. This observation was consistent with results for magenta. Light green did not exhibit fully functioning hydrophobic barriers even at $300 \mu \mathrm{m}$ nominal width, most likely due to the high ratio of yellow which showed relatively small spreading. Similar results were obtained for the three test solutions (Terasil Blue, Acid Green and Orange $\mathrm{G}$ ), indicating that the dyes had no significant influence on the experiment.

For further experiments, all $\mu \mathrm{PAD}$ patterns were designed in magenta with $W_{\mathrm{N}}=200 \mu \mathrm{m}$. Magenta was the most suitable

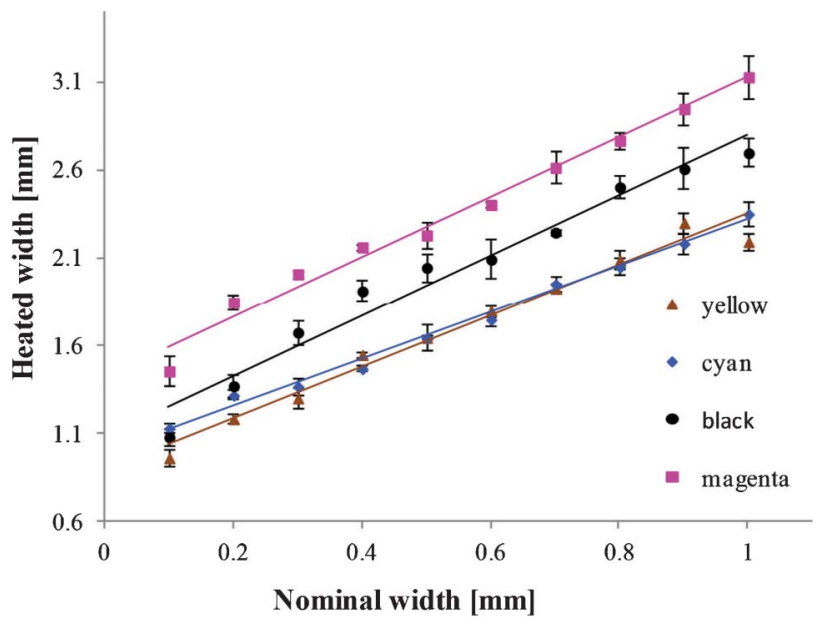

Fig. 4 Nominal widths against widths after heating for the CMYK colours present in the four different solid inks used in the ColorQube 8870 colour printer. colour for the generation of reliable hydrophobic barriers for two reasons. Firstly, it showed the largest spreading when compared to cyan, yellow and black. Secondly, it is a single colour and possible inconsistencies caused by colour mixing could therefore be excluded. The four ColorQube 8870 solid

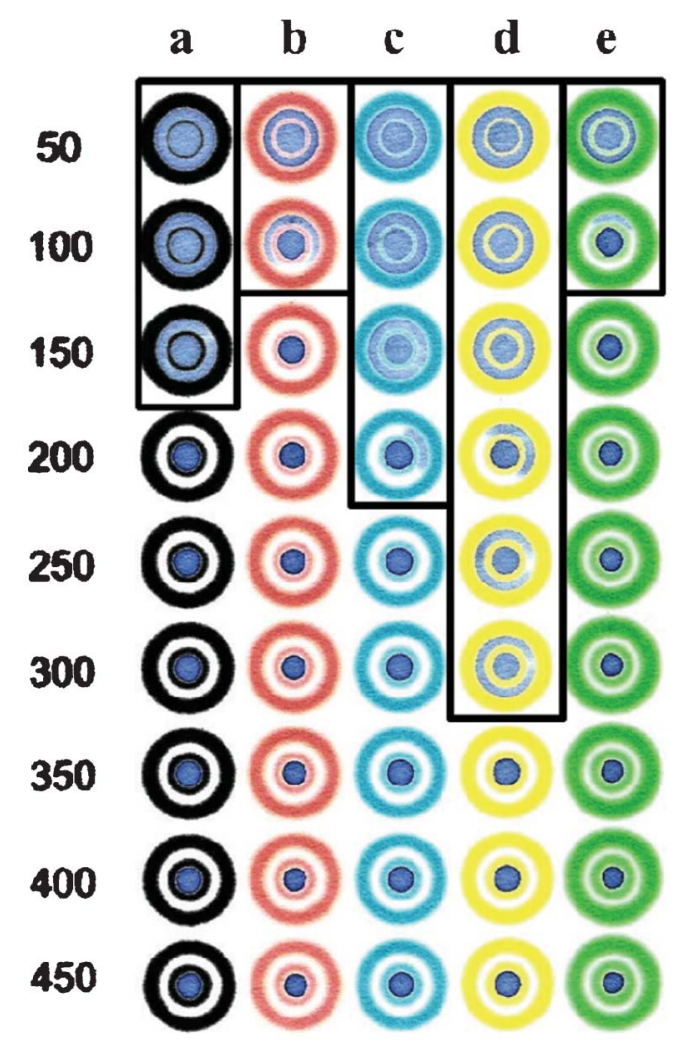

Fig. 5 Coloured hydrophobic circles in black (a), magenta (b), cyan (c), yellow (d) and green (e) with nominal widths of the inner circle from 50 to $450 \mu \mathrm{m}$ with increments of $50 \mu \mathrm{m}$ (see left column) generated under the same conditions as in Fig. 3. All circles are filled with $5 \mu \mathrm{L}$ of a $1 \mathrm{mg} \mathrm{mL}^{-1}$ Terasil Blue aqueous solution. The marked areas highlight inner circles which are not reliable hydrophobic barriers as the solution does not stay within them. Image taken under visible light. ${ }^{39}$ 
inks are made of polyethylene and fatty amine waxes (50$60 \%)$, resin (10-20\%) and dye ( $\leqslant$ than $5 \%$ ), although the exact composition of each one of the four solid inks is proprietary information. Two different batches of inks were tested and the results were similar implying that at least for this printer magenta produces better hydrophobic barriers than the other standard colours.

\section{Application: explosive detection by fluorescence quenching}

Pyrene is a flat polycyclic aromatic hydrocarbon soluble in several different organic solvents, however just sparingly soluble in water $\left(0.135 \mathrm{mg} \mathrm{\textrm {L } ^ { - 1 }}\right) .{ }^{43}$ Pyrene was selected as the fluorophore based on its excellent relative fluorescence power of $0.345^{43}$ and its known quenching in the presence of explosive material. ${ }^{44}$ However, the pyrene application to the $\mu$ PADs was found to be problematic.

The water solubility and therefore pyrene concentration in water was too low for fluorescence detection. On the other hand, the hydrophobicity of the wax channels restricted the application of pyrene in organic solvents to the $\mu$ PADs. It was assumed that less polar solvent molecules than water, e.g. methanol, ethanol, propanol and acetonitrile (solvents usually used to dissolve explosives), can slightly interact with the fatty acids in the solid ink allowing the passage of the solvents. Two reported approaches to increase the water solubility of pyrene, i.e. increasing the temperature ${ }^{45}$ and adding surfactants, ${ }^{46}$ were both tested without success. Increasing the temperature did not result in a satisfactory pyrene concentration and the surfactant property of SDS lowered the surface tension of water so that the pyrene-SDS solution did not stay compartmentalized in the barrier.

The possibility of first dissolving the pyrene in an organic solvent and then mixing the organic solution with water and applying the dispersion to the hydrophobic channels was investigated. To find the required ratio of water : organic solvent for $\mu \mathrm{PAD}$ application, coloured (Terasil Blue) dispersions with different ratios (from 10:90 to 100:0) were deposited on circles printed in magenta. The results can be seen in Fig. 6.

For methanol : water (column A), a ratio of $80: 20$ respectively displayed satisfactory hydrophilic properties for the application on $\mu$ PADs. An ethanol : water mixture required a $50 \%$ water ratio, whilst a propanol : water mixture required a $90 \%$ water ratio. Therefore each incorporation of a methylene group in the alcohol molecule reduced the capacity of retention of the solution on the $\mu \mathrm{PAD}$ by $30-40 \%$. Acetonitrile showed satisfactory hydrophilic properties when mixed with water in a $50: 50$ ratio similar to ethanol. This sequence of alcohols (methanol, ethanol, propanol) was expected and can be explained by the different solubility of alcohols in water caused by the different sizes of the nonpolar carbon chain.

As organic solvents were able to penetrate the wax barriers, experiments were conducted to test whether this process resulted in any change of the barrier quality. Hexane (hydrophobic solvent) and methanol (hydrophilic solvent) were separately pipetted $(5 \mu \mathrm{L})$ in the $\mu \mathrm{PAD}$ circles and allowed to dry. Afterwards, coloured solvent : water mixtures were deposited on the $\mu \mathrm{PAD}$ (same design as in Fig. 6). Treated

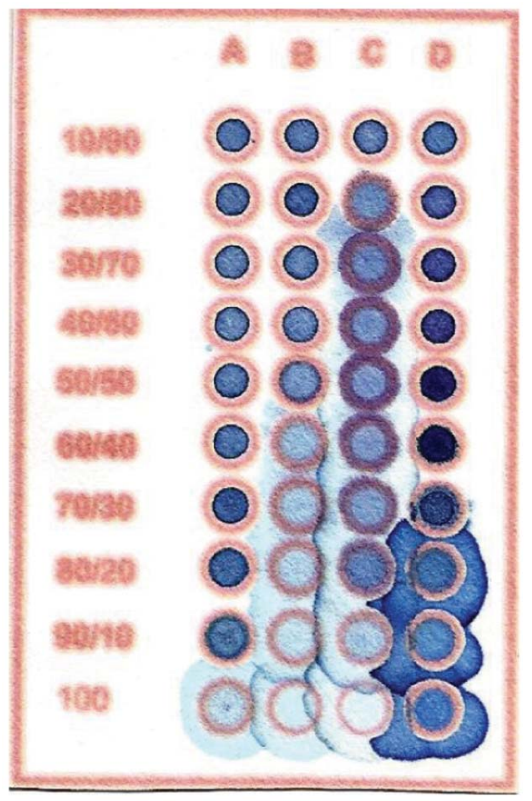

Fig. 6 Column A-methanol : water mixture, B-ethanol : water mixture, C-propanol : water mixture and D-acetonitrile : water mixture. Ratios of organic solvents: water are given in the left column starting from $10 \%$ organic solvent at the top to $100 \%$ at the bottom. Picture is taken with visible light. All solutions are coloured with $1 \mathrm{mg} \mathrm{mL}^{-1}$ Terasil Blue.

(with hexane or methanol) and untreated (previous experiment, Fig. 6) barriers showed similar hydrophilic character. It was therefore concluded, that organic solvents do not have noticeable influence on the quality of the wax barriers. Methanol : water $(80: 20)$ was chosen as the solvent system for all experiments as the water percentage was the smallest in comparison to the other organic solvents and the pyrene concentration and thus fluorescence relatively high. Different pyrene concentrations were tested from 0.1 to $1 \mathrm{mg} \mathrm{mL}^{-1}$ for their fluorescence under the VSC 6000 instrument. A concentration of $0.5 \mathrm{mg} \mathrm{mL}^{-1}$ pyrene in methanol : water $(80: 20)$ was determined as optimal concentration for further experiments. While lower concentrations $\left(0.1\right.$ and $\left.0.25 \mathrm{mg} \mathrm{mL}^{-1}\right)$ did not show satisfactory fluorescence, higher concentrations

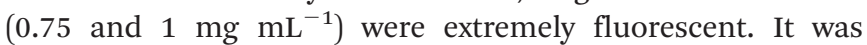
observed that high fluorescence could exacerbate the detection of explosives in low concentrations as no change in the fluorescence was noticed. One $\mu \mathrm{L}$ of the optimised pyrene solution (0.5 mg mL $\mathrm{mL}^{-1}$ in $80: 20$ methanol : water) was deposited in the hydrophobic barrier of the $\mu \mathrm{PAD}$. Under UV light, the $\mu \mathrm{PAD}$ fluoresced, while in contact with explosives the fluorescence was quenched.

Ten different explosives were tested for fluorescence quenching of pyrene with the VSC 6000 instrument. The choice of explosives was based on covering a range of classes of explosives such as nitrate esters (PETN), nitro aromatics (TNT, 2,4-DNT, 4-A-2,6-DNT, 4-NT, DNB) and nitro amines (RDX, tetryl). One $\mu \mathrm{L}$ of the explosive solution (1000 ppm) was deposited within the hydrophobic barriers of the $\mu \mathrm{PAD}$. All explosives gave positive results as shown in Fig. 7 . 


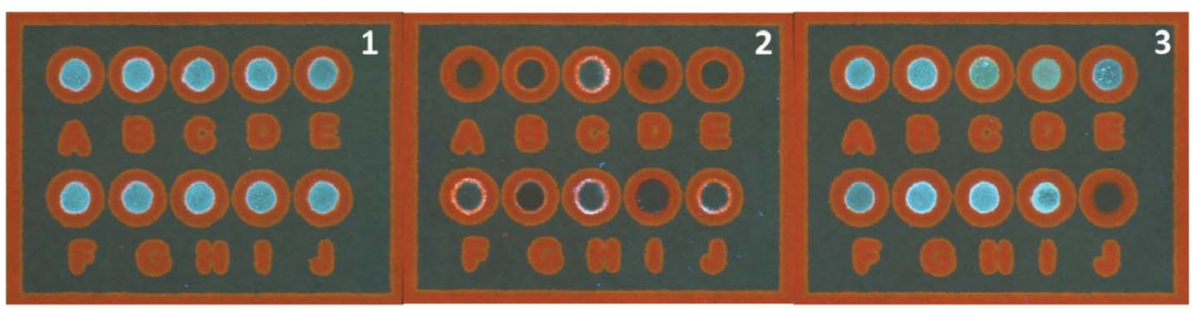

Fig. 71 - $\mu \mathrm{PAD}$ with $1 \mu \mathrm{L}$ of $0.5 \mathrm{mg} \mathrm{mL}^{-1}$ pyrene solution in methanol: water $(80: 20)$ on circles with $5 \mathrm{~mm}$ diameter generated under the same conditions as in Fig. 3. 2- Same $\mu \mathrm{PAD}$ after the deposition of $1 \mu \mathrm{L}$ of 10 different explosives ( $\mathrm{A}: 1,3,5$-trinitrobenzene, $\mathrm{B}: 1,3$-dinitrobenzene, $\mathrm{C}$ : nitrobenzene, D: 2,4,6-trinitrotoluene, E: 2,4-dinitrotoluene, F: 4-nitrotoluene, G: 4-amino-2,6-dinitrotoluene, H: RDX, I: tetryl, J: PETN) demonstrating fluorescence quenching. 3- $\mu$ PAD generated under the same conditions with nine different non-explosive substances and one explosive (A: negative control, B: water, C: milk, D: coffee, E: tea, F: coke, G: beer, H: wine, I: Mylanta Antacid Dual Action and J: 2,4,6-trinitrotoluene (positive control)).

The $\mu \mathrm{PAD}$ proved to be suitable for the detection of nitrate esters, nitro aromatics, and nitro amines.

As can be seen in Fig. 7-2 the affinity between explosive and pyrene depends of the chemical composition of the explosive. TNT (D) for example displayed higher fluorescence quenching in comparison with 4-NT (F). The detection limits of the ten explosives tested (Fig. 7-2) using the VSC 6000 instrument were found to be between 100 and $600 \mathrm{ppm}$.

Other explosives including picric acid and dinitrotoluene isomers were also tested positive using this method, but are not presented here.

It was observed, that solvents used to prepare standard solutions, such as acetonitrile and methanol, can decrease the fluorescence of pyrene. However, this decrease was extremely limited and could be easily distinguished from fluorescence quenching caused by the deposition of explosives.

The selectivity of the method was investigated by examining eight randomly selected substances (water, milk, coffee, tea, coke, beer, wine, Mylanta Antacid Dual Action) for their ability to quench the fluorescence of the $\mu \mathrm{PAD}$ device compared to a negative and positive control. All tests for the eight randomly selected substances were negative (Fig. 7-3). Preliminary data indicated that the selectivity of the technique was good, with no false positives detected.

\section{Portable explosive detector prototype}

A small, portable instrument was built for the field detection of explosives. This instrument incorporated a $365 \mathrm{~nm}$ UV LED as a light source and a photodiode as a light sensor. The use of a high power LED was necessary to ensure sufficient excitation of pyrene. The detection cell, where the $\mu \mathrm{PAD}$ was inserted, had a gap of $0.5 \mathrm{~mm}$ between the light source and sensor. When the UV LED was turned on, the ultraviolet light (365 nm) excited the pyrene on the $\mu \mathrm{PAD}$ which emitted visible light. This light was captured by the photodiode sensor at $500 \mathrm{~nm}$ on the other side of the $\mu \mathrm{PAD}$ (Fig. 8). The sensitivity of the photosensor was relatively low at $365 \mathrm{~nm}$, which was important in order to avoid interferences with the UV light that passes through the paper. When a new $\mu$ PAD device was inserted in the instrument, calibration was achieved by presenting a circle with only pyrene to the sensor and adjusting the potentiometer R2 (see Fig. 1) until the green LED was turned on. The green light signalled that no explosives were present on the $\mu \mathrm{PAD}$. If explosives were on the $\mu \mathrm{PAD}$, fluorescence quenching shifted the wavelength which was usually emitted by the pyrene and a red light flashed to alert the analyst (see D2 - Fig. 1).

The instrument was tested by sampling a $10 \mathrm{~cm} \times 10 \mathrm{~cm}$ bench surface which had been thoroughly cleaned and was free from any explosive material. A cotton swab moistened with methanol : water ( $80: 20)$ was used as sampling device. After sampling, the swab was gently pressed on top of a circle on the $\mu \mathrm{PAD}$. The $\mu \mathrm{PAD}$ was then inserted into the instrument which displayed a green light, indicating no detection of explosives. After re-sampling the bench surface homogeneously spiked with TNT ( $3 \mu \mathrm{L}$ of $1000 \mathrm{ppm}$ solution) and exposing the respective $\mu \mathrm{PAD}$, the instrument displayed a red flashing light indicating the detection of explosives.

A preliminary test of all ten explosives which had previously given positive results using the VSC 6000 instrument, also resulted in a positive response with the portable prototype and no false-positives were detected. These results demonstrate the suitability of the prototype $\mu \mathrm{PAD}$ reader to explosive screening. Fig. 8 illustrates the portable explosive detector prototype.

Optimisation. As the sensor has a $5 \mathrm{~mm}$ diameter, this diameter was chosen as an ideal size for the circle printed on the $\mu \mathrm{PAD}$. Three different pyrene concentrations, i.e. $0.10 \mathrm{mg}$ $\mathrm{mL}^{-1}, 0.25 \mathrm{mg} \mathrm{mL}^{-1}$ and $0.50 \mathrm{mg} \mathrm{mL}^{-1}$ were tested. Optimal performance was found when $1 \mu \mathrm{L}$ of $0.25 \mathrm{mg} \mathrm{mL}^{-1}$ pyrene solution was deposited on the $\mu \mathrm{PAD}$.

\section{Conclusion}

An innovative, rapid, cost-effective and simple screening method for the detection of explosives based on $\mu$ PADs has been developed. Micropads were created by printing on a filter paper using a FujiXerox ColorQube 8870 wax printer and heating the paper at $150{ }^{\circ} \mathrm{C}$ for $5 \mathrm{~min}$ with a heat press. Magenta was found to be the most suitable colour for generating hydrophobic barriers with a smaller nominal width, i.e. $120 \mu \mathrm{m}$, to create a fully functioning hydrophobic barrier than other colours. As the created $\mu$ PADs are not affected by temperatures under $60{ }^{\circ} \mathrm{C}$, storage for field-based use is promising. 

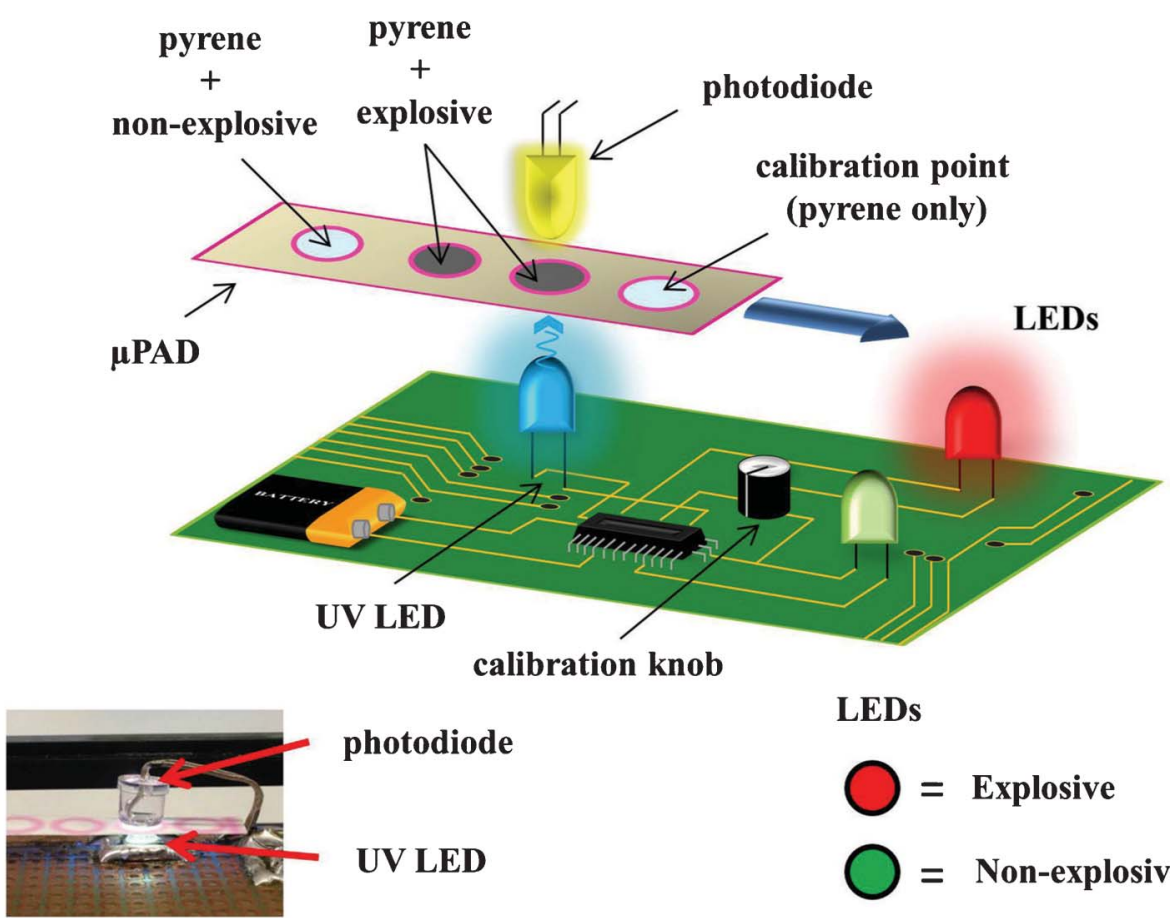

LEDs

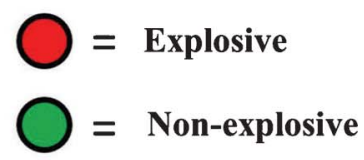

Fig. 8 Illustration of the portable explosive detector prototype. The first step (not shown) includes inserting the calibration point between the UV LED and the photodiode and turning the calibration knob until the green LED flashes. The second step (displayed in the Figure) shows the detection of explosives on the $\mu$ PAD (red LED flashes).

Table 1 Minimum detectable masses of 10 explosives using the prototype explosive detector and an optimized. $\mu$ PAD $\left(1 \mu \mathrm{L} 0.25 \mathrm{mg} \mathrm{mL}^{-1} \mathrm{pyrene}, 5 \mathrm{~mm}\right.$ diameter circle)

\begin{tabular}{llllllllll}
\hline TNT & $2,4-D N T$ & $4-A-2,6-D N T$ & $4-N T$ & TNB & 1,3-DNB & NB & RDX & PETN & Tetryl \\
\hline $0.2 \mu \mathrm{g}$ & $0.5 \mu \mathrm{g}$ & $0.3 \mu \mathrm{g}$ & $0.7 \mu \mathrm{g}$ & $0.1 \mu \mathrm{g}$ & $0.7 \mu \mathrm{g}$ & $0.9 \mu \mathrm{g}$ & $0.8 \mu \mathrm{g}$ & $0.8 \mu \mathrm{g}$ & $0.4 \mu \mathrm{g}$
\end{tabular}

Pyrene was successfully deposited on the $\mu$ PADs by dissolving it in an organic solvent (methanol) and adding water to the solution to create a $80: 20$ ratio dispersion. The detection of ten different explosives representing nitrate esters, nitro aromatics and nitro amines with the VSC 6000 has been demonstrated with limits of detection between 100 and $600 \mathrm{ppm}$, thus in the nanogram range. The method is able to detect explosives in aqueous or organic solutions. No false positives were detected indicating a good selectivity of the developed method. However, this study needs to be expanded to further examine the selectivity of the method and identify any potential false positives to therefore increase the evidential value of the technique.

A portable battery-operated instrument prototype was built to enable the field-based detection of explosives. The light source and optical sensor were based on the use of an UV-LED and a photosensor and met the requirements for high reliability, portability, low power and low cost. The sensor emits a red light alarming signal when fluorescence quenching is detected. All ten explosives tested with the VSC 6000 were also detected by the prototype. By decreasing the concentration of pyrene to $0.25 \mathrm{mg} \mathrm{mL} \mathrm{m}^{-1}$, the minimum detectable masses for the ten tested explosives were in between 0.1 to 0.9 micrograms (Table 1). The developed method has the potential for field-based application of explosive detection for environmental and law enforcement purposes. Further research will focus on improving the sensitivity and robustness of the developed method and the portable prototype, which will result in its casework applicability. This can be achieved in two ways: (1) optimising the instrument or (2) optimising the $\mu \mathrm{PAD}$.

\section{Acknowledgements}

This research was financially supported by the Commonwealth of Australia through the National Security Science and Technology Centre within the Defence Science and Technology Organisation. 


\section{References}

1 E. Holmgren, et al., Determination and characterization of organic explosives using porous graphitic carbon and liquid chromatography-atmospheric pressure chemical ionization mass spectrometry, J. Chromatogr., A, 2005, 1099(1-2), 127-135.

2 O. Vigneau and X. Machuron-Mandard, A LC-MS method allowing the analysis of HMX and RDX present at the picogram level in natural aqueous samples without a concentration step, Talanta, 2009, 77(5), 1609-1613.

3 E. Erçağ, et al., Rapid detection of nitroaromatic and nitramine explosives on chromatographic paper and their reflectometric sensing on PVC tablets, Talanta, 2011, 85(4), 2226-2232.

4 P. R. Gauger, et al., Explosives detection in soil using a field-portable continuous flow immunosensor, J. Hazard. Mater., 2001, 83(1-2), 51-63.

5 R. F. Spalding and J. W. Fulton, Groundwater munition residues and nitrate near Grand Island, Nebraska, USA, J. Contam. Hydrol., 1988, 2(2), 139-153.

6 A. M. Jiménez and M. J. Navas, Chemiluminescence detection systems for the analysis of explosives, J. Hazard. Mater., 2004, 106(1), 1-8.

7 J. Wang, et al., Single-Channel Microchip for Fast Screening and Detailed Identification of Nitroaromatic Explosives or Organophosphate Nerve Agents, Anal. Chem., 2002, 74(5), 1187-1191.

8 B. Paull, et al., Rapid Screening of Selected Organic Explosives by High Performance Liquid Chromatography Using Reversed-Phase Monolithic Columns, J. Forensic Sci., 2004, 49(6), 1-6.

9 J. A. Mathis and B. R. McCord, The analysis of high explosives by liquid chromatography/electrospray ionization mass spectrometry: multiplexed detection of negative ion adducts, Rapid Commun. Mass Spectrom., 2005, 19(2), 99-104.

10 J. M. F. Douse, Trace analysis of explosives in handswab extracts using Amberlite XAD-7 porous polymer beads, silica capillary column gas chromatography with electroncapture detection and thin-layer chromatography, $J$. Chromatogr., A, 1982, 234(2), 415-425.

11 S. Casamento, et al., Optimization of the Separation of Organic Explosives by Capillary Electrophoresis with Artificial Neural Networks, J. Forensic Sci., 2003, 48(5), 1-9.

12 J. M. Sylvia, et al., Surface-Enhanced Raman Detection of 2,4-Dinitrotoluene Impurity Vapor as a Marker To Locate Landmines, Anal. Chem., 2000, 72(23), 5834-5840.

13 R. D. Luggar, et al., Multivariate analysis of statistically poor EDXRD spectra for the detection of concealed explosives, X-Ray Spectrom., 1998, 27(2), 87-94.

14 R. G. Ewing, et al., A critical review of ion mobility spectrometry for the detection of explosives and explosive related compounds, Talanta, 2001, 54(3), 515-529.

15 M. E. Sigman and C.-Y. Ma, Detection Limits for GC/MS Analysis of Organic Explosives, J. Forensic Sci., 2001, 46(1), 6-11.

16 K. Håkansson, et al., Low-mass ions observed in plasma desorption mass spectrometry of high explosives, J. Mass Spectrom., 2000, 35(3), 337-346.
17 S. J. Toal and W. C. Trogler, Polymer sensors for nitroaromatic explosives detection, J. Mater. Chem., 2006, 16(28), 2871-2883.

18 W. J. Buttner, et al., In situ detection of trinitrotoluene and other nitrated explosives in soils, Anal. Chim. Acta, 1997, 341(1), 63-71.

19 M. E. Germain and M. J. Knapp, Optical explosives detection: from color changes to fluorescence turn-on, Chem. Soc. Rev., 2009, 38(9), 2543-2555.

20 A. W. Kusterbeck, et al., A continuous flow immunoassay for rapid and sensitive detection of small molecules, $J$. Immunol. Methods, 1990, 135(1-2), 191-197.

21 S. Y. Rabbany, et al., Trace detection of explosives using a membrane-based displacement immunoassay, J. Immunol. Methods, 2000, 246(1-2), 69-77.

22 G. P. Anderson and E. R. Goldman, TNT detection using llama antibodies and a two-step competitive fluid array immunoassay, J. Immunol. Methods, 2008, 339(1), 47-54.

23 E. R. Goldman, et al., Analysis of aqueous 2,4,6-trinitrotoluene (TNT) using a fluorescent displacement immunoassay, Anal. Bioanal. Chem., 2003, 375(4), 471-475.

24 E. R. Goldman, et al., Detection of 2,4,6-Trinitrotoluene in Environmental Samples Using a Homogeneous Fluoroimmunoassay, Environ. Sci. Technol., 2003, 37(20), 4733-4736.

25 R. T. Medary, Inexpensive, rapid field screening test for 2,4,6-trinitrotoluene in soil, Anal. Chim. Acta, 1992, 258(2), 341-346.

26 Y. Jiang, et al., A Simple Assay for Direct Colorimetric Visualization of Trinitrotoluene at Picomolar Levels Using Gold Nanoparticles, Angew. Chem., Int. Ed., 2008, 47(45), 8601-8604.

27 J.-S. Yang and T. M. Swager, Fluorescent Porous Polymer Films as TNT Chemosensors: Electronic and Structural Effects, J. Am. Chem. Soc., 1998, 120(46), 11864-11873.

28 S. W. Thomas, G. D. Joly and T. M. Swager, Chemical Sensors Based on Amplifying Fluorescent Conjugated Polymers, Chem. Rev., 2007, 107(4), 1339-1386.

29 P. Anzenbacher, et al., Iptycene-Based Fluorescent Sensors for Nitroaromatics and TNT, Chem.-Eur. J., 2012, 18(40), 12712-12718.

$30 \mathrm{Y}$. Xin, et al., A portable and autonomous multichannel fluorescence detector for on-line and in situ explosive detection in aqueous phase, Lab Chip, 2012, 12(22), 4821-4828.

31 S. Zhang, et al., Fluorescent film sensors based on SAMs of pyrene derivatives for detecting nitroaromatics in aqueous solutions, Spectrochim. Acta, Part A, 2012, 97, 31-7.

32 A. W. Martinez, et al., FLASH: A rapid method for prototyping paper-based microfluidic devices, Lab Chip, 2008, 8(12), 2146-2150.

$33 \mathrm{~J}$. Nie, et al., Low-Cost Fabrication of Paper-Based Microfluidic Devices by One-Step Plotting, Anal. Chem., 2012, 84(15), 6331-6335.

34 E. M. Fenton, et al., Multiplex Lateral-Flow Test Strips Fabricated by Two-Dimensional Shaping, ACS Appl. Mater. Interfaces, 2008, 1(1), 124-129.

35 X. Li, et al., Paper-Based Microfluidic Devices by Plasma Treatment, Anal. Chem., 2008, 80(23), 9131-9134.

$36 \mathrm{X} . \mathrm{Li}$, et al., Fabrication of paper-based microfluidic sensors by printing, Colloids Surf., B, 2010, 76(2), 564-570. 
37 K. Abe, K. Suzuki and D. Citterio, Inkjet-Printed Microfluidic Multianalyte Chemical Sensing Paper, Anal. Chem., 2008, 80(18), 6928-6934.

38 K. Abe, et al., Inkjet-printed paperfluidic immuno-chemical sensing device, Anal. Bioanal. Chem., 2010, 398(2), 885-893.

39 E. Carrilho, A. W. Martinez and G. M. Whitesides, Understanding Wax Printing: A Simple Micropatterning Process for Paper-Based Microfluidics, Anal. Chem., 2009, 81(16), 7091-7095.

$40 \mathrm{Y}$. Lu, et al., Rapid prototyping of paper-based microfluidics with wax for low-cost, portable bioassay, Electrophoresis, 2009, 30(9), 1497-1500.

41 Y. Lu, et al., Fabrication and Characterization of PaperBased Microfluidics Prepared in Nitrocellulose Membrane by Wax Printing, Anal. Chem., 2010, 82(1), 329-335.

42 A. W. Martinez, et al., Diagnostics for the Developing World: Microfluidic Paper-Based Analytical Devices, Anal. Chem., 2010, 82(1), 3-10.
43 M. S. Meaney and V. L. McGuffin, Investigation of common fluorophores for the detection of nitrated explosives by fluorescence quenching, Anal. Chim. Acta, 2008, 610(1), 57-67.

44 K.-S. Focsaneanu and J. C. Scaiano, Potential analytical applications of differential fluorescence quenching: pyrene monomer and excimer emissions as sensors for electron deficient molecules, Photochem. Photobiol. Sci., 2005, 4(10), 817-821.

45 R. D. Wauchope and F. W. Getzen, Temperature dependence of solubilities in water and heats of fusion of solid aromatic hydrocarbons, J. Chem. Eng. Data, 1972, 17(1), 38-41.

46 L. Z. Zhu and C. T. Chiou, Water solubility enhancements of pyrene by single and mixed surfactant solutions, $J$. Environ. Sci. (China), 2001, 13(4), 491-496. 